OPEN ACCESS

Citation: A. Moscaritolo, A. Dupuy, P. Ratinaud, C. Z. Gaudron. (2021) Vécu de familles monoparentales avec jeunes enfants durant le premier confinement lié à la COVID-19 en France. Rief 18, 2: pp. 201-216. doi: https://doi.org/10.36253/rief10179

Copyright: (C) 2021 A. Moscaritolo, A. Dupuy, P. Ratinaud, C. Z. Gaudron This is an open access, peer-reviewed article published by Firenze University Press (https://oaj.fupress.net/ index.php/rief) and distributed under the terms of the Creative Commons Attribution License, which permits unrestricted use, distribution, and reproduction in any medium, provided the original author and source are credited.

Data Availability Statement: All relevant data are within the paper and its Supporting Information files.

Competing Interests: The Author(s) declare(s) no conflict of interest.

\section{Vécu de familles monoparentales avec jeunes enfants durant le premier confinement lié à la COVID-19 en France}

\author{
Alice Moscaritolo', Anne Dupuy ${ }^{2}$, Pierre Ratinaud ${ }^{3}$, \\ Chantal Zaouche Gaudron ${ }^{4,5}$
}

Résumé

En France, comme dans de nombreux pays, les mesures imposées lors de la pandémie de COVID-19 ont bouleversé le vécu des familles. Parmi elles, le vécu des familles monoparentales avec jeunes enfants semble singulier. L’article réside dans l'analyse des réponses à un questionnaire en ligne diffusé pendant le premier confinement, auquel certains de ces foyers se sont soumis, en mettant en perspective le vécu exprimé et les conditions de vie. Les données confirment une situation de plus grande fragilité de ces familles comparativement aux autres de l'enquête en révélant une distribution inégale des conséquences du confinement au regard des niveaux d'étude, de ressources et de conditions de logement. Pour les parents "solo", principalement des mères, la charge affective, éducative et de soins accrue s'est heurtée aux pressions ressenties concernant la façon de s'occuper de ses enfants, ainsi quà des perceptions moins satisfaisantes de l'exercice du rôle parental. Pour autant, la plupart des relations parent-enfant se sont renforcées. L'investissement de mesures de protection hors gestes barrière témoigne d'une sensibilité accrue face à la maladie. Le peu de mention faite de l'entourage social est particulièrement flagrant.

Mots-cles: COVID-19, confinement, familles monoparentales, jeunes enfants, inégalités.

${ }^{1}$ Post-doctorante en Sciences de l'éducation et de la formation au Groupement d'Intérêt Scientifique (GIS) "Bébé petite Enfance en COntextes (BECO)" - Université Fédérale de Toulouse Midi-Pyrénées, et jeune chercheure à l'Unité Mixte de Recherche "Education Formation Travail Savoirs" (EFTS, MA 122).

${ }^{2}$ Maîtresse de Conférences en Sociologie à l'Université de Toulouse - Jean Jaurès, et membre du Centre de Recherche sur le Travail, l'Organisation, le Pouvoir (CERTOP, UMR-CNRS 5044).

${ }^{3}$ Maître de Conférences en Sciences de l'éducation et de la formation à l'Université de Toulouse - Jean Jaurès, et membre du Laboratoire d'Études et de Recherches Appliquées en Sciences Sociales (LERASS, Université de Toulouse III - Paul Sabatier)

${ }^{4}$ Professeure de Psychologie de l'enfant à Université de Toulouse - Jean Jaurès, Directrice du Groupement d'Intérêt Scientifique (GIS) "Bébé petite Enfance en COntextes (BECO)", Directrice adjointe de l'Institut Fédératif d'Etudes et de Recherches Interdisciplinaires Santé Société (IFERISS), et membre du Laboratoire Interdisciplinaire Solidarités, Sociétés, Territoires (LISST, UMR 5193).

${ }^{5}$ Cet article est le fruit du travail commun des Auteurs (sauf indication contraire, toutes les notes de bas de page sont dorénavant des Auteurs, N.D.L.R.). 
Abstract

In Francia, come in molti Paesi, le misure imposte durante la pandemia da COVID-19 hanno sconvolto il vissuto quotidiano delle famiglie, in particolare quelle monoparentali con figli piccoli. L'articolo riporta in sintesi l'analisi delle risposte a un sondaggio online effettuato durante il primo lockdown a cui hanno partecipato alcune di queste famiglie, mettendo in relazione l'esperienza vissuta in tale contesto con le loro condizioni di vita. I dati confermano che queste famiglie si trovano in una situazione di maggior fragilità rispetto alle altre coinvolte nell'indagine. Ciò appare evidente dalla diversa gravità dell'impatto che il lockdown ha avuto, per quanto riguarda i livelli di istruzione, le risorse e le condizioni abitative. Per i genitori single, principalmente le madri, l'aumento del carico emotivo, educativo e di cura è stato posto a confronto con la pressione percepita, relativa alla cura dei figli, nonché con sensazioni di minor soddisfazione circa la propria genitorialità. Tuttavia, la maggior parte delle relazioni genitore-figlio è diventata più forte. L'adozione di misure protettive diverse dai meri gesti di distanziamento sociale mostra una maggiore sensibilità nei confronti di questa malattia. Colpisce particolarmente la scarsità, nelle risposte, di riferimenti al ruolo della rete sociale.

Parole chiave: COVID-19, lockdown, famiglie monoparentali, bambini, diseguaglianze.

\section{Introduction}

En France, dès la mi-mars 2020, tous et toutes ont été confrontés au même mot d'ordre "Restez chez vous"; il s'agit bien d'un message, conçu en raison de l'urgence sanitaire, qui a conduit les familles, comme chaque citoyen et citoyenne, à expérimenter d'autres formes de relations, d'interactions, d'activités. Le confinement, instauré afin de limiter le plus possible les échanges sociaux pouvant accélérer la diffusion de la COVID-19, a induit ainsi des formes encore inédites de vie au sein du foyer. D'une certaine façon, les familles ont subi l'injonction de se replier sur elles-mêmes, de par l'organisation sanitaire instituée $e^{6}$, en limitant, le plus possible, les échanges quotidiens aux seuls membres de la famille confinés dans un même logement. La socialisation familiale s'est trouvée profondément modifiée en raison de la fermeture des crèches (ou autres structures de la petite enfance), des structures scolaires ou des services périscolaires et de l'impossibilité à avoir recours à une garde grand-parentale.

Compte tenu des difficultés et souffrances engendrées par la pandémie de COVID-19 pour de nombreuses familles, ce contexte sans précédent dans notre pays a semblé nécessaire à étudier en particulier pour les familles ayant des enfants de six ans et moins, la petite enfance étant une période de construction et de vulnérabilité. Aussi, avons-nous ${ }^{7}$ jugé important d'analyser ce que les parents et les jeunes enfants ont vécu, ressenti, mobilisé durant cette période de confinement. Le nombre peu important de travaux en Sciences Humaines et Sociales sur la prime enfance et sur l'univers familial pendant ce contexte rend singulière notre analyse.

La crise sanitaire peut accroître les inégalités sociales entre enfants, notamment pour ce qui concerne le suivi scolaire (Bonnéry, Douat, 2020) et les formes de loisirs proposés. Les parents ne disposent pas tous des mêmes ressources (Zaouche Gaudron, 2017) et dispositions

\footnotetext{
${ }^{6}$ Les sorties sur le territoire national français ont été limitées, sous peine d'amende, à une heure par jour dans un rayon d'un kilomètre, sauf pour des raisons définies par décret.

${ }^{7}$ Pour le Consortium “COV-JEUNENFANT”: C.Z. Gaudron (Dir.), J.-C. Basson, C. Bouilhac, I. Claudet, N. Collomb, C. Delpierre, A. Dupuy, E. Gaborit, I. Glorieux, V. Katkoff, M. Kelly-Irving, V. Larrosa, P. Marchand, C. Mennesson, A. Moscaritolo, S. Pinel-Jacquemin, P. Ratinaud, J. Renard, T.-N. Willig.
} 
pour assurer le suivi éducatif ou scolaire de leurs enfants et organiser leur temps libre et leur occupation professionnelle (Bessière et al., 2020; Lambert et al., 2020).

Nous focalisons, ainsi dans cet article, notre attention sur les familles monoparentales, estimant qu'à la situation "ordinaire" déjà complexe du parent isolé se rajoutaient de nouvelles conditions de vie (éducation et garde des jeunes enfants, travail et télétravail) liées à la pandémie, complexifiant le vécu de chaque membre du foyer. Aux potentielles difficultés financières, s'ajoutait, par ailleurs, l'absence du soutien familial, juridique et social lors du confinement (Zhou et al., 2020). Nous avons souhaité explorer la question des inégalités par l'intermédiaire de ces conditions inédites, potentiellement révélatrices ou amplificatrices de données connues avant la pandémie, ici, la monoparentalité.

\section{Méthodologie}

Une méthode mixte a été privilégiée. Une analyse quantitative a d'abord permis de décrire l'échantillon, de dégager les relations entre les variables et de vérifier les hypothèses. Dans un deuxième temps, l'analyse qualitative du corpus discursif est venue éclairer ces premiers résultats.

La méthode quantitative n'a pu se réaliser avec des quotas. En effet, les contraintes spécifiques du confinement orientant le protocole d'enquête vers le recours à un mode d'autosaisie en ligne du questionnaire, un biais d'échantillonnage concernant les propriétés sociales des enquêtés est saillant concernant l'échantillon de notre enquête pouvant et acceptant de témoigner de leur vécu. Cela signifie que la taille de l'échantillon est réduite et que celle concernant les foyers monoparentaux l'est d'autant plus. Cela indique également que sur un certain nombre de critères, les caractéristiques des répondants diffèrent de celles de l'ensemble de la population à laquelle elles appartiennent. Cette limite inhérente à l'échantillonnage à partir d'une enquête autosaisie est soulevée par d'autres enquêtes analogues ${ }^{8}$. De même, nous pouvons faire l'hypothèse que le temps nécessaire pour répondre à l'enquête a davantage impacté les familles monoparentales de par leur impossibilité de déléguer ou partager les tâches incompressibles et dégager un temps suffisant pour le faire.

\subsection{Outil utilisé}

Entre le 28 avril 2020 et le 29 mai $2020^{9}, 490$ personnes $^{10}$ résidant en France ont répondu à un questionnaire en ligne diffusé aux familles ayant au moins un enfant entre zéro et six ans via un site internet dédié ${ }^{11}$ et différents réseaux des partenaires de l'étude (académiques, professionnels, informels, social media). Le questionnaire est composé de 69 questions (51 à choix multiple, 18 ouvertes), qui se répartissent de la façon suivante : informations générales (items 1 à 18); l'expression libre du parent (item 19), le vécu du confinement (item 20 à 26); l'expression libre de l'enfant, lorsque possible (item 27); les activités et la vie quotidienne pendant le confinement (items 28 à 46); le logement pendant le confinement (items 47 à 58); l'emploi

\footnotetext{
${ }^{8}$ Nous pouvons citer à ce propos les enquêtes "VIe en Confinement" (VICO), CNRS, LISST, EHESS, (https:// vico.hypotheses.org/94/94; date de dernière consultation: 16.04.21) et "Effect of COVID-related COntainment in ChildreN” (E-COCCON), PUPH, Hôpital des Enfants, UMR 1027-Equity.

${ }^{9}$ Les dates officielles du confinement en France ont été du 17 mars au 11 mai 2020. Le questionnaire étant resté en ligne au-delà du 11 mai, quelques-uns ont été renseignés après le 11 mai.

${ }^{10}$ Après nettoyage des données initiales.

${ }^{11}$ https://pinel-jacquemin.wixsite.com/covjenfant; date de dernière consultation: 22.12.20.
} 
depuis le début du confinement (items 59 à 68); un commentaire libre sur ce qui a semblé important aux participants (enfants et parents, item 69). Les répondants ont pu préciser leurs réponses dans des espaces de commentaire prévus à cet effet pour 21 questions.

La réalisation de cette étude a été validée par le service Délégué à la Protection des Données pour la mise en conformité du protocole de collecte avec le Règlement Général sur la Protection des Données (RGPD-CNRS) ainsi que par le comité d'Ethique sur les Recherches (CER, numéro 2020-290) de l'Université Fédérale de Toulouse Midi- Pyrénées.

\subsection{Echantillon et méthodes d'analyse}

Sur la population de 490 répondants, l'échantillon des familles monoparentales (FM, $\mathrm{n}=$ 36) est composé de parents sans conjoint cohabitant et vivant avec un ou plusieurs enfants ${ }^{12}$, dont a minima un enfant âgé de six ans ou moins.

Une description de l'échantillon des FM par un tri à plat a servi de base pour une première comparaison avec la population de l'étude (PE). A l'aide du logiciel StataCorp. 2019 (version 16.1), les corrélations entre les deux variables "familles monoparentales" versus "biparentales" et les variables sociodémographiques ont été soumises au test de significativité statistique du $\mathrm{chi}^{2}$ et au test exact de Fisher*, les valeurs-p globales discriminant les résultats selon un seuil de significativité de 0,05 .

Des analyses bivariées ont également vérifié les relations entre les deux variables citées supra et sept scores créés à partir des items du questionnaire (voir Annexe 1).

L'analyse quantitative des données recueillies est complétée par une analyse qualitative de thématisation continue (Paillé, Mucchielli, 2016) des expressions libres (items 19, 27 et 69).

\section{Principaux résultats}

\subsection{Données socio-descriptives}

L'échantillon des familles monoparentales $(\mathrm{FM}, \mathrm{n}=36)$ compte 32 mères $^{13}$ et quatre pères, la répartition de genre étant équivalente à la distribution dans la population d'étude (PE). La proportion réduite de notre échantillon (7\% de la $\mathrm{PE})$ par rapport au taux national français ( $21 \%$ en 2018 d'après l'INSEE ${ }^{14}$ ) peut s'expliquer par le jeune âge des enfants de la population enquêtée, les séparations conjugales intervenant plus tardivement après la naissance d'un enfant. En France, trois millions d'enfants, soit plus d'un enfant sur cinq, sont élevés dans une famille monoparentale, et dans $84 \%$ des cas par leur mère ${ }^{15} .37 \%$ de ces enfants occupent un logement social ${ }^{16}$. Mais ces données nationales se situent tous âges confondus, de la naissance jusqu’à la majorité, soit dix-huit ans, ce qui rend difficile la comparaison avec notre échantillon.

${ }^{12}$ D'après la définition donnée par l'Institut national de la statistique et des études économiques (INSEE), https:/www.insee.fr/fr/metadonnees/definition/c1936; date de dernière consultation: 07.02.21.

${ }^{13} \mathrm{Au}$ vu du fait que les parents sont très majoritairement des femmes, nous allons par la suite utiliser le féminin pour caractériser les sujets de l'étude.

${ }^{14} \mathrm{https} / /$ www.insee.fr/fr/statistiques/4285341; date de dernière consultation: 12.04.21.

${ }^{15} \mathrm{https} / / / \mathrm{www}$. insee.fr/fr/statistiques/4277630?sommaire=431829; date de dernière consultation: 12.04.21.

${ }^{16}$ Voir la note 8. 
Au moment de l'enquête, $31 \mathrm{FM}$ résident en zone "verte" ${ }^{17}$, dont 25 en Occitanie, troisième région de France en termes de nombre de familles monoparentales (une famille sur quatre), dont un tiers sont pauvres ${ }^{18}$, proportion analogue au taux national (en France un tiers des FM vivent sous le seuil de pauvreté; Vanderschelden, 2014). Les répondantes de l'échantillon FM comptent très majoritairement un seul enfant $(72 \%$ vs $35 \%$ des foyers biparentaux, $\mathrm{p}<0,0001)$. Lorsqu'il y a un deuxième enfant confiné, le plus souvent, il a entre cinq et six ans $(\mathrm{p}<0,0001)$ et fréquente l'école maternelle $\left(\mathrm{p}=0,008^{*}\right)$.

Principalement employées, ouvrières et aides à domicile (31\% vs $20 \%$ des familles biparentales, FB) et deux fois plus à ne pas avoir d'activité professionnelle (19\% vs $9 \%)$, les répondantes en FM ont un niveau d'études inférieur à la population d'étude, la catégorie socio-professionnelle et le diplôme des répondantes étant significativement liés aux variables "FM" et "FB" ( $\mathrm{p}$ $=0,046^{*}$ et $\mathrm{p}=0,012^{*}$, respectivement).

Les revenus mensuels de l'échantillon des FM sont plus faibles : pour $78 \%$ inférieurs à 2 500 euros nets (contre 13\% des FB, $\mathrm{p}<0,0001$ ), quatre fois plus de foyers se situent en dessous du seuil de pauvreté ${ }^{19}$ ( $42 \%$ vs $10 \%$ de la PE).

Presque le double de foyers FM se situent dans le tercile ${ }^{20}$ de revenus le plus bas $(61 \%$ vs $34 \%$ dans la PE) et présentent un revenu médian inférieur dans chaque tercile. Les ressources ont été davantage réduites lors du confinement: $31 \%$ a connu une diminution significative des revenus, $19 \%$ une mise au chômage (contre $19 \%$ et $8 \%$ dans la PE, respectivement). La plupart a le sentiment de ne pas être «du tout à l'aise» financièrement ( $53 \%$ vs $17 \%$ des FB, $\mathrm{p}$ $<0,0001$ ), ainsi qu'un tiers des cadres de l'échantillon (trois sur neuf), dont deux se situent dans le premier tercile de revenu par unité de consommation et un en dessous du seuil de pauvreté.

Un peu plus de la moitié des FB disposent de plus de quatre pièces (53\%), contre seulement $19 \%$ des FM ( $p<0,0001)$, qui résident davantage en habitat mixte ou en immeuble $(64 \% v s$ $35 \%$ des $\mathrm{FB}, \mathrm{p}=0,001)$, ainsi qu'en appartement $\left(58 \%\right.$ vs $\left.33 \%, \mathrm{p}=0,011^{*}\right)$. Néanmoins, au vu de la composition du foyer, $81 \%$ des FM contre $56 \%$ des FB disposent de plus d'une pièce par personne $\left(\mathrm{p}=0,009^{*}\right)$.

\subsection{Thèmes et sous-thèmes discursifs}

L'analyse qualitative de thématisation continue a dégagé quatre grands thèmes, répartis en 17 sous-thèmes pour les verbatims des parents $(n=36)$ et en neuf sous-thèmes pour les verbatims d'enfants retranscrits par les parents $(\mathrm{n}=17)$ (voir le Tableau 1).

${ }^{17}$ Le Ministère des Solidarités et de la Santé a défini trois zones à risque au 3 mai 2020: 46 départements "verts", 18 départements "oranges" et 32 départements "rouges".

${ }^{18} \mathrm{https} / /$ www.insee.fr/fr/statistiques/4241048\#titre-bloc-12; date de dernière consultation: 12.04.21.

${ }^{19}$ Le seuil de pauvreté au seuil de $60 \%$ correspond à un revenu mensuel inférieur à 1.063 Euros, https://www. insee.fr/fr/statistiques/2499760; date de dernière consultation: 16.04.21.

${ }^{20}$ La variable "revenu par unités de consommation (uc)" a été créée à partir de l'échelle de l'OCDE qui attribue 1 "uc" au premier adulte du ménage, 0,5 "uc" aux autres personnes de 14 ans ou plus et 0,3 "uc" aux enfants de moins de 14 ans. 
Verbatims des parents ( $\mathrm{n}=36 ; 183$ syntagmes)

Item 19: «Nous vous invitons ici à vous exprimer le plus librement possible sur cette période "inordinaire” pour mieux comprendre comment vous et vos jeunes enfants vivez cette période de confinement (au niveau des activités, du jeu, des règles, de l'utilisation des écrans, de l'alimentation, de la scolarité, de l'éducation au sens large, etc.), ce que vous éprouvez (comme émotions, peurs, colères, exaspérations, lassitudes, mais aussi plaisirs, joies, amusements, par exemple, etc.) et ce que vous pensez».

Item 69: Commentaire libre

Verbatims des enfants ( $\mathrm{n}=17 ; 44$ syntagmes)

Item 27 : «Si l'un de vos enfants de moins de 6 ans le souhaite (ou plusieurs), nous l'(les)invitons ici à répondre le plus librement possible à la question suivante: "Par rapport à ta vie en ce moment, qu'est-ce que tu pourrais en dire?"”.

\begin{tabular}{|c|c|c|c|c|}
\hline \multirow{2}{*}{$\begin{array}{l}17 \text { sous- } \\
\text { thèmes } \\
\text { Parents }\end{array}$} & \multicolumn{4}{|c|}{4 THEMES } \\
\hline & $\begin{array}{c}\text { Conditions de vie } \\
(56,6 \% \text { des syntagmes })\end{array}$ & $\begin{array}{l}\text { Ressenti } \\
(27,5 \%)\end{array}$ & $\begin{array}{l}\text { Relations } \\
(11 \%)\end{array}$ & $\begin{array}{l}\text { COVID-19 } \\
(5 \%)\end{array}$ \\
\hline \multirow{9}{*}{$\begin{array}{l}9 \text { sous- } \\
\text { thèmes } \\
\text { Enfants }\end{array}$} & $\begin{array}{l}\text { Loisir (19 répondantes) } \\
\text { ( } 7 \text { enfants) }\end{array}$ & $\begin{array}{l}\text { Sentiments positifs ( } 22) \\
\text { (8 enfants) }\end{array}$ & $\begin{array}{l}\text { Lien social (11) } \\
\text { (9 enfants) }\end{array}$ & $\begin{array}{l}\text { Contraintes (6) } \\
\text { (3 enfants) }\end{array}$ \\
\hline & $\begin{array}{l}\text { Ecole (18) } \\
\text { (6 enfants) }\end{array}$ & $\begin{array}{l}\text { Sentiments négatifs ( } 22 \text { ) } \\
\text { ( } 3 \text { enfants) }\end{array}$ & $\begin{array}{l}\text { L'autre parent (6) } \\
\text { (4 enfants) }\end{array}$ & $\begin{array}{l}\text { Maladie (3) } \\
\text { (3 enfants) }\end{array}$ \\
\hline & Médias (17) & $\begin{array}{l}\text { Ambivalence } \\
\text { émotionnelle (6) }\end{array}$ & $\begin{array}{l}\text { Echanges avec les } \\
\text { enfants (4) }\end{array}$ & \\
\hline & $\begin{array}{l}\text { Conciliation vie familiale- } \\
\text { vie professionnelle (14) }\end{array}$ & & & \\
\hline & Gestion du temps (12) & & & \\
\hline & $\begin{array}{l}\text { Alimentation (8) } \\
\text { (1 enfant) }\end{array}$ & & & \\
\hline & Tâches domestiques (6) & & & \\
\hline & Sommeil (5) & & & \\
\hline & Espaces de vie (4) & & & \\
\hline
\end{tabular}

Tableau 1 - Thèmes et sous-thèmes issus de l'analyse qualitative

\subsection{Organiser "l'entre-soi"}

Le thème occupant la plus grande part des expressions libres des répondantes est relatif à la description de leurs conditions de vie de "confinées", sans doute en raison de la formulation de l'item 19 qui a pu les orienter en ce sens. En effet, un peu plus de la moitié des syntagmes analysés (57\%) en font mention. Néanmoins, le sous-thème récurrent qui réfère à la conciliation vie familiale-vie professionnelle mentionné par 14 des 36 répondantes, n'était pas suggéré dans l'énoncé.

Par ailleurs, quatre fois plus de mères "solo" en arrêt, en congés ou inactives ont repris le travail pendant le confinement ( $8 \%$ vs $2 \%$ des FB). Toutes les 14 répondantes ont fait état des problèmes rencontrés, qualifiant la conciliation de «difficile», «compliquée», voire «impossible»: 
"Période complexe où il faut tout concilier. Le télétravail [...] est une solution pour l'employeur et pour maintenir un salaire mais en termes de confort de vie et de sérénité c'est un vrai calvaire [...]. Je suis épuisée de tout concilien (une mère d'un enfant, 35 ans, profession intermédiaire).

Le surmenage en est une conséquence qui sape l'énergie: "Etre sur tous les fronts fatigue énormément» (une employée de 38 ans, mère de trois enfants); "Je me suis mise beaucoup de pression et j'ai craqué au bout de trois semaines» (une employée de 41 ans, mère de deux enfants); ce témoignage se retrouve exprimé par l'un des quatre pères de l'échantillon: "Papa seul en télétravail qui se transforme en instituteur, nounou, homme de ménage, cuistot trois fois par joun (un cadre de 36 ans, père de deux enfants).

Dans les syntagmes analysés, la gestion du temps de la vie "confinée" suit de près les perceptions sur les conditions de vie (12 répondantes). Les 10 occurrences du terme «rythme» sont associées à la mise en place d'une organisation bénéfique au foyer, soit avec l'établissement de règles, déjà présentes ou adaptées, soit en s'accordant plus de souplesse: «C’était indispensable pour nous d'avoir des repères temporels, un rythme régulier. J'ai l'impression qu'on a plutôt bien vécu cette période, grâce à cette visibilité sur nos journées [...] il y avait de la place pour la liberté de chacun aussi, et des règles de vie de la maison inchangées» (mère d'un enfant, 32 ans, sans activité professionnelle).

Sur les huit syntagmes relatifs au sous-thème de l'alimentation, sept ont une acception positive, en association avec l'articulation du temps quotidien, ou bien en tant qu'occasion de partage ou d'amélioration de la qualité des repas, «exacerbant l'importance déjà accordée à l'alimentation» (41 ans, mère d'un enfant, profession intermédiaire), avec pour certaines une influence sur l'image que l'on a de soi: "Je rejoue à fond mon rôle de mère, avec plus de plats et de vrais repas à la maison» (mère de deux enfants, 48 ans, cadre).

La mention des tâches domestiques (six syntagmes) fait le plus souvent appel à la participation accrue des enfants, vue par certaines comme occasion d'apprentissage et d'autonomisation, même si deux répondantes mentionnent la charge accrue. Lorsqu'il y a référence explicite au lieu de confinement (quatre syntagmes, deux cadres et deux employées), l'on mentionne la présence d'un extérieur (balcon, terrasse, jardin, etc.), décrit en termes de "privilège» et de «chance». En effet, le taux des FM dotées d'espace extérieur privatif est légèrement inférieur à la PE $(80,5 \%$ vs $89 \%$ ) mais supérieur à la moyenne nationale ${ }^{21}$.

\subsection{Les relations, entre manque et entretien}

Le thème des relations est décliné en trois sous-thèmes. En premier lieu apparaît le manque de lien social pour les enfants, rapporté par huit mères sur les 11 répondants le mentionnant. Ce sous-thème est, par ailleurs, le premier présent dans les verbatims des enfants, cité davantage que par leurs parents (neuf enfants sur 17). Les enfants s'expriment en termes de manque des amis d'abord (cinq syntagmes), de la maîtresse (quatre), de la famille (trois), des grands-parents (un) et des voisins (un) ensuite.

Néanmoins, le confinement a amené, pour certain.es, d'autres formes de socialisation: un père (en profession libérale, 40 ans) relate que par des «jeux avec des enfants sans contact (cabane, courses en imitant les animaux, vélo...) [prénom de sa fille, 4 ans] s'est faite beaucoup d'amies»;

\footnotetext{
${ }^{21}$ En France, les espaces de vie de 30\% des FM ne disposent pas d'extérieur, contre 14\% des couples avec enfants (Bugeja-Bloch, Crepin, 2020).
} 
une mère (cadre, 44 ans, un enfant) rapporte l'expérience d'un confinement à trois familles dans une maison de vacances, où les enfants ont eu "la chance de vivre dans une sorte de fratrie, en communauté [...]. Deux des enfants sont en famille monoparentale et ont donc découvert la vie avec un papa et une maman».

Le sous-thème "relation à l'autre parent" est mentionné dans six témoignages d'adultes (cinq mères et un père) et quatre d'enfants. A une exception près dans chaque groupe, il l'est en des termes positifs, évoquant le rapprochement entre ex partenaires et l'organisation mise en place pour gérer la garde des enfants dans un contexte inhabituel, ainsi que les nécessaires compromis et arrangements.

Le sous-thème des "échanges avec les enfants" concerne quatre témoignages d'accompagnement de l'expérience du confinement par la parole: "Nous discutons beaucoup. La raison de ne pas retourner à la crèche est clairement identifiée» (père d'un enfant, 44 ans, profession intermédiaire); "J'ai [...] fait en sorte de lui expliquer les choses avec des mots simples mais vrais, et de ne pas amener la peur dans notre maison» (mère d'un enfant, 32 ans, sans activité professionnelle).

\subsection{Le domaine de la parentalité}

La charge parentale horaire assumée en FM a été plus importante que celle observée dans la PE: pour accompagner le travail scolaire, plus de quatre heures quotidiennes $(17 \%$ contre $6 \%$ dans la PE); pour réaliser des activités de loisirs et culturelles, plus de six heures pour $31 \%$, contre $16 \%$.

Dans leurs expressions libres, un peu plus de la moitié des FM (19, dont deux pères) mentionnent les loisirs, la moitié est concernée par l'école (18, dont deux pères), et une part importante (17, dont trois pères) cite les médias, surtout en association avec la gestion des écrans. Les trois sous-thèmes liés aux activités éducatives et de loisirs ("école", "loisirs", "médias") sont les plus cités après les deux sous-thèmes liés aux émotions. Dans 17 des 19 témoignages énumérant les loisirs, les activités sont un moment agréable et remplissent les journées en contribuant à une temporalité rythmée dont les bénéfices ont été mentionnés: "Le matin, lecture ensemble, gym ensemble, petits jeux autonomes (beaucoup d'inventions d'histoire) et sortie. La fin d'après-midi et début de soirée: un jeu ou une activité ensemble, parfois une autre sortie, douche ou bain, télé-apéro. Lecture avant de dormin» (44 ans, père d'un enfant, profession intermédiaire). Les temps d'extérieur, limités par les restrictions en vigueur, ont été recherchés par les familles (13 témoignages sur 19). Ce besoin d'extérieur ressort surtout des témoignages des enfants : cité en troisième place (sept témoignages sur 17), après le lien social et les sentiments positifs, le sous-thème du loisir est entièrement consacré au manque ressenti des sorties d'avant le confinement.

La charge pédagogique assurée pendant le confinement a entraîné des difficultés pour plus de la moitié des familles relatant des tâches scolaires effectuées (11 sur 18). Les tâches scolaires à mettre en place ont demandé une configuration inédite à laquelle les parents n'avaient pas été préparés avant la pandémie: «On ne simprovise pas enseignant, comme semble le penser l'Education nationale» (une mère d'un enfant, 43 ans, sans activité professionnelle). Faire école à la maison a été une activité «compliquée», source de «conflits» et de "pression». Certaines familles ont réduit les attentes quand d'autres ont abandonné cette charge éducative supplémentaire: "J'ai lâché l'enseignement à l'école. [...] Nous ne faisons que quelques exercices ponctuels. Je ne voulais pas prendre le risque d'entrer en conflit avec mes enfants en cette période» (une mère de deux enfants, 41 ans, employée).

Seulement trois mères sur les 36 parents ont fait mention d'un lien maintenu avec les maîtresses de leurs enfants scolarisés en maternelle, dans deux cas s'agissant de vidéos envoyées aux parents. La difficulté mentionnée par trois parents a été la dissonance cognitive-affective 
causée par la conciliation du travail scolaire avec un milieu de vie habituellement identifié comme distinct: "Il y avait là au départ comme une incompréhension, un malaise. Quelque chose n'était pas à sa place» (mère d'un enfant, 41 ans, profession intermédiaire); "L'école lui donnait certains repères symboliques qu'il n’a pas à la maison» (mère d'un enfant, 34 ans, sans activité professionnelle). Sur les six enfants ayant mentionné l'école, les deux enfants ayant témoigné de leurs difficultés ont des parents ayant également exprimé un ressenti négatif à ce sujet. Le manque motivationnel de se mettre au travail scolaire au sein du domicile s'allie dans les discours des enfants à la «hâte» de retourner à l'école d'avant le confinement: «L'école à la maison c'est bien mais j'ai pas envie de faire l'école alors je m'en veux. Mais j'aime la vraie école en vrai, je sais que tu fais de ton mieux pour me faire la vraie école» (5 ans).

L'augmentation du temps des écrans est une question soulevée par un peu plus de la moitié des familles (neuf sur 17), même si elle reste contenue et raisonnable d'après la plupart d'entre elles. Tout en posant des limites définies au préalable avec les jeunes enfants, l'écart dans l'utilisation des activités numériques durant le confinement est expliqué en termes de «règles plus souples».

Si l'on considère le regard porté sur l'exercice du rôle parental dans le contexte du confinement, mères et pères en FM se sont perçus moins armés pour stimuler ou éduquer leurs enfants ( $50 \%$ vs $34 \%$ de la population d'étude) et moins compétents face aux activités éducatives (33\% vs 20\%). Un besoin d'informations concernant l'éducation de leurs enfants (règles, interdictions) a été davantage exprimé par rapport aux familles biparentales ( $31 \%$ vs 17\% des FB, p = 0,041).

La pression exercée par les médias sur la façon de s'occuper des enfants pendant la période du confinement a été plus aigüe pour les parents en FM (22\% vs 10\% des FB). Aussi, le score de pression ressentie concernant la façon de s'occuper de ses enfants présente une corrélation très significative avec les variables "FM" et "FB" ( $p=0,006)$. En effet, $44 \%$ des FM ressentent entre une et deux pressions fortes, contre $32 \%$ des $\mathrm{FB}$, et $22 \%$ plus de deux, contre $10 \%$.

Les relations parents-enfants se sont davantage modifiées pendant le confinement $(\mathrm{p}=0,002)$. Si les tensions se sont exacerbées dans certaines familles (17\% vs $10 \%$ des FB), les liens se sont renforcés dans la plupart des cas (53\% vs 28\%): «Bilan de ce confinement: les liens de fratrie se sont resserrés, les liens maternels aussi. Ça fait du bien que de voir son ou ses enfants évoluer, apprendre, participer, appréhender..." (une de mère de trois enfants, 38 ans, employée); «notre foyer sen est trouvé resserré et nos relations sont très profondes» (un père de deux enfants, 36 ans, cadre).

\subsection{Santé physique et psychique}

Les personnes ayant rencontré des problèmes de santé autres que la COVID-19 pendant le confinement sont presque le double dans les FM ( $47 \%$ vs $27 \%$ de la PE). Particulièrement saillante dans l'échantillon des FM est l'attention accordée aux mesures de protection contre l'épidémie hors gestes barrière ${ }^{22}(\mathrm{p}=0,022): 50 \%$ des FM lavent leurs propres vêtements portés en extérieur ( vs $27 \%$ dans la PE) et $47 \%$ ceux de leurs enfants (vs $24 \%$ ). La désinfection des poignées de porte et des interrupteurs est aussi surinvestie, surtout en tant que mesure de protection des enfants $(36 \%$ vs 26\%). Cette attention accrue est en partie explicable par la nécessité de sortir davantage: mis à part les courses, les familles monoparentales sortent plusieurs fois par jour, tous les jours ou presque ( $28 \%$ vs $13,5 \%)$. Aussi, les lavages supplémentaires et

\footnotetext{
${ }^{22}$ Les gestes barrière figurant dans notre questionnaire sont ceux rattachés aux prescriptions sanitaires du gouvernement français à l'époque du premier confinement (se laver les mains, utiliser du gel hydroalcoolique, respecter un mètre de distance avec les autres), plus le fait de porter un masque.
} 
les produits désinfectants ajoutent des coûts à des foyers déjà moins nantis. Le prix élevé des masques durant le premier confinement pourrait en partie expliquer le moindre recours à cette mesure de protection ( $50 \%$ vs 59\%), les difficultés d'approvisionnement et les consignes incertaines étant d'autres freins possibles à son utilisation.

La proportion d'enfants faisant mention explicite du thème de la COVID-19 est plus importante que celle retrouvée auprès des adultes (six enfants sur $17 v$ seuf adultes sur 36), avec une envie de trouver une solution au problème: "C'est un vilain microbe qui tue les gens...Il ménerve ce virus... J'ai une idée je suis docteur je fabrique un médicament» (3 ans) ; "J'ai pas peur du virus moi, je vais prendre mon marteau et la scie et je vais l'attaquer comme ça il va avoir peur et il va partin» (3 ans). Chez les répondantes, la présence de l'épidémie est davantage exprimée en termes des contraintes qui en découlent (six syntagmes): le port du masque, la limitation des sorties, les pénuries, retrouvées également dans les verbatims de trois enfants. Aussi, les conséquences du confinement sur la santé des enfants sont rapportées par cinq parents en termes de troubles du sommeil.

D'un point de vue psychique, les familles monoparentales ont vécu le confinement un peu plus difficilement que la population d'étude, les répondantes en FM se disant un peu moins en forme $(39 \%$ vs $50 \%)$ et un peu moins heureuses (64\% vs $74 \%)$. Elles sont plus nombreuses à ressentir que les choses n'allaient "presque jamais» ou «jamais» (36\% vs 19\%) comme elles le voulaient. En effet, le score de difficulté ressentie depuis le début du confinement révèle une différence statistiquement significative $(\mathrm{p}=0,015)$ entre les variables "FM" et "FB": un peu plus de la moitié des FM (53\% vs 33\% des FB) a «souvent» rencontré des difficultés à contrôler les choses importantes de leur vie et à gérer les problèmes personnels.

La prégnance du discours émotionnel est signifiée par le fait que les deux sous-thèmes s’y référant soient les plus cités. Le ressenti des familles est néanmoins partagé: les syntagmes exprimant des émotions négatives et ceux qui relatent des émotions positives sont en nombre égal (22 pour chaque sous-thème). Parmi les ressentis négatifs, de l'inquiétude, de l'angoisse, de l'incertitude, de l'anxiété, du refus, de la peur, de l'incompréhension, de la frustration, qui parfois prennent la forme d'un appel au secours: "Je me sens totalement perdue et noyée» (une cadre mère d'un enfant, 45 ans); "Il m'arrive de ressentir de grands moments de solitude et de tristesse» (une mère d'un enfant, 38 ans, sans activité professionnelle); "Je vis cette situation comme épuisante, éprouvante à en arriver au point de ne plus supporter d'entendre mon enfant» (une mère d'un enfant, 31 ans, en formation). Les ressentis négatifs sont beaucoup moins présents dans les discours des enfants (trois verbatims) et expriment principalement une gêne ou de l'ennui: "C'est injuste d'être confinée. C'est assez embêtant» (5 ans).

Sur un tout autre registre, le confinement est arrivé pour certaines familles comme une pause inattendue dans un quotidien souvent sous tension: "Dans les premiers jours [...] un sentiment de soulagement, une pause forcée qui était bienvenue» (une cadre de 40 ans, mère de deux enfants). Pour quelques-unes, il s'agit d'une opportunité de se réapproprier sa vie de famille: "C'est une occasion inespérée de passer plus de temps avec mes enfants [...] Je redécouvre mes enfants, qui sont du coup plus calmes, car moi plus zen... et on a du temps pour passer le temps!!!! (une cadre de 48 ans, mère de deux enfants). Pour d'autres, cette suspension de l'ordinaire a produit des réflexions plus profondes sur les raisons d'être et d'agir : "Cette période a été révélatrice $d u$ potentiel de ressources internes de chacun dans cette absence de contact avec l'entourage habituellement soutenant» (41 ans, mère d'un enfant, profession intermédiaire).

Pour les enfants, le ressenti positif apparaît comme deuxième sous-thème (huit témoignages): "J'aime rester à la maison, parce que maman est là» (5 ans); "Je me sens bien. Ni triste ni super joyeux» (5 ans); "mon fils m'a dit qu'il ressentait l'émotion de la rose et de la jaune (amour et joie du livre la couleur des émotions)» (4 ans). 
Dans un peu plus de la moitié des témoignages mentionnant un registre émotionnel (12 sur 22), les sentiments positifs et négatifs s'entremêlent. Six répondantes font état d'une ambivalence émotionnelle, penchant positif et négatif étant inextricables: "Emotionnellement c'est un peu le grand 8, avec un combat quotidien pour ne pas flanchern (une cadre de 40 ans, mère de deux enfants); "Par phase dispo, ou aspirée et épuisée par leurs sollicitation [...]. Mais bien moins stressée que l'avant-COVID!» (une mère de deux enfants, 40 ans, profession intermédiaire). Dans quatre cas sur six, cette ambivalence est associée à des syntagmes de ressentis négatifs.

\section{Discussion}

Les analyses effectuées sur les variables viennent étayer d'autres recherches soulignant la fragilité "ordinaire" et quotidienne de nombreuses familles monoparentales (Bugeja-Bloch, Crepin, 2020; Neyrand, Rossi, 2007; Vanderschelden, 2014; Zaouche Gaudron, 2017).

Néanmoins, elles ont révélé la présence d'effets spécifiques au contexte du confinement: les scores de "pression ressentie" et de "difficulté ressentie", ainsi que le besoin d'informations concernant l'éducation des enfants sont des variables très fortement ou fortement liées à la monoparentalité.

Les recherches qui, à ce jour, identifient la problématique de la monoparentalité en lien avec la pandémie de COVID-19 mettent en avant des facteurs de vulnérabilité accrus qui corroborent certains résultats de notre étude. Marchetti et collègues (2020) ont inclus le fait d'être célibataire, ainsi que d'avoir des enfants en bas âge parmi les facteurs prédictifs d'un plus grand épuisement lié au rôle de parent; dans l'étude de Tso et collègues (2020) sur la population des familles de Hong Kong dont plus de douze mille avec enfants âgés de deux à cinq ans, le fait de vivre avec un seul parent figure parmi les facteurs prédisposant à des risques psychosociaux plus élevés chez les enfants. La recherche d'Almeida et collègues (2020) montre que les disparités entre les sexes déjà présentes en temps ordinaires ont été accentuées pour les parents isolés, la plupart du temps des mères, car les femmes sont responsables de manière disproportionnée de la majeure partie des tâches domestiques et des pratiques de soins prodigués aux enfants.

Nous constatons, comme dans d'autres enquêtes (KIHSA, citée par Choi et collègues, 2020), que les familles monoparentales les plus précaires, en dessous du seuil de pauvreté, dont le parent ne travaille pas ou est à temps partiel, sont susceptibles de présenter un vécu encore plus complexe de la vie "confinée": les 12 foyers les plus fragiles d'un point de vue socio-économique de notre échantillon (10 mères et deux pères) mentionnent moins de sentiments positifs (six témoignages) et davantage de sentiments négatifs (neuf). Trois parents sur huit indiquant le travail scolaire font référence à une charge supplémentaire occasionnée par cette activité, à mettre en relation avec le temps plus important alloué par les familles populaires pour s'acquitter de cette tâche lors du confinement (Thierry et al., 2021). Autre fait marquant, aucune mention n'est faite de l'autre parent, absence encore plus flagrante dans les sept témoignages de leurs enfants.

Ces derniers mentionnent proportionnellement moins les loisirs (deux sur sept), plus l'école (trois sur sept) et plus souvent la maladie et les contraintes liées au confinement (deux sur sept).

La lecture transversale des verbatims issue de l'analyse thématique du corpus discursif a induit une moindre visibilité de certaines sous-catégories de familles monoparentales, dont celles avec fratrie, ou comportant un père isolé, voire de catégorie socio-professionnelle supérieure.

Pour toutes les familles monoparentales de notre étude, l'absence d'une mention aux relations entretenues avec l'extérieur du foyer est tout aussi remarquable. Ce fait rejoint les conclusions d'Okabe-Miyamoto et collègues (2021) sur l'influence prépondérante de la présence d'un partenaire au sein du foyer dans l'entretien des relations sociales pendant le confinement. Autrement dit, le manque de 
relations sociales est exacerbé pour les familles monoparentales laissant supposer un "entre soi” encore plus prégnant que dans les foyers biparentaux, voire un isolement social important.

Malgré la petite taille de l'échantillon de familles monoparentales qui a servi de base aux analyses et aux réflexions présentées ici, qui ne peuvent pas prêter à généralisation, les tendances mises en exergue peuvent orienter d'autres études de plus grande envergure.

\section{Conclusion}

Lors du premier confinement les familles monoparentales enquêtées ont connu une situation stressante, conditionnée par des conditions socio-économiques préexistantes, qui risquent de s'aggraver encore avec la crise socio-économique qui se profile pour nombre d'entre elles. La charge éducative, affective et de soins, les pressions ressenties et une perception moins satisfaisante de l'exercice du rôle parental ont pu avoir des répercussions sur leur état de santé physique et psychique. Tout en résidant principalement en zone épidémique "verte", elles sont sensibles face au risque de contracter le virus et ressentent, sans doute plus que d'autres, la nécessité de préserver leur santé. En raison du manque de relais possible pour l'ensemble des activités quotidiennes, de l'absence de l'autre parent, l'investissement dans les mesures de protection hors gestes barrière renforcées démontre une inquiétude supplémentaire.

Enfin, il est aussi fort probable que la fermeture des structures d'accueil et scolaires, l'impossibilité d'avoir recours à une garde grand-parentale ou toute autre forme de bricolages (Modak, Zaouche Gaudron, Séraphin, 2021; Zaouche Gaudron, Boyer, 2021) a rendu la situation encore plus complexe à gérer pour les familles monoparentales en raison de ce déficit de soutien familial et/ou social durant le confinement. Les structures scolaires semblent avoir contribué très peu à entretenir à distance ce lien social dont les familles monoparentales avec de jeunes enfants ont ressenti un grand manque. N'oublions pas que les enfants ont évoqué, avant tout, le manque des relations, mises en suspens durant le premier confinement alors qu'elles jouent un rôle dans le bien-être des jeunes enfants.

Compte tenu de la situation sans précédent engendrée par la pandémie et des contraintes particulières auxquelles ces foyers sont confrontés, qu'elles soient socio-économiques ou dues à l'absence de soutien social, il est nécessaire que les pouvoirs publics portent une attention toute particulière aux familles composées de parents seuls, le plus souvent des mères, avec des enfants en bas âge, d'autant plus que la pandémie est encore active sur notre territoire national et ailleurs.

\section{Remerciements}

Les résultats présentés dans cet article s'appuient sur l'Enquête "COV-JEUNENFANT", financée par l'Agence Nationale de la Recherche, le Conseil régional Occitanie, la Caisse Nationale des Allocations Familiales, la Caisse d'Allocations Familiales 31, le Conseil départemental de la Haute Garonne. Nous remercions Amalia Martinez (GIS BECO - UFTMiP) pour les traitements statistiques des données quantitatives qu'elle a réalisés, ainsi que l'Université Fédérale Toulouse Midi-Pyrénées pour son soutien institutionnel et technique. Plusieurs questions sont reprises d'un questionnaire plus général formulé par des enseignants-chercheurs CNRS que nous remercions vivement ${ }^{23}$. Nos remerciements vont aux familles pour leur participation à notre étude.

\section{(Aucun conflit d'intérêt)}

\footnotetext{
${ }^{23} \mathrm{https} / / /$ sms.univ-tlse2.fr/parcours-de-vie-et-reseaux-personnels-/enquete-relations-sociales-et-solidarites-en-periode-de-confinement-confinement--729547.kjsp?RH=ACCUEIL_SMS; date de dernière consultation: 16.04.21.
} 
Annexes

\begin{tabular}{|c|c|}
\hline \multicolumn{2}{|c|}{ Score Difficulté ressentie durant le confinement (item n. 26) } \\
\hline Classes & $\begin{array}{l}2 \text { classes : «jamais» et «presque jamais» (codées “0”), «parfois» et «très sou- } \\
\text { vent» (codées “1”) }\end{array}$ \\
\hline Enoncés & $\begin{array}{l}\text { - Vous a-t-il semblé difficile de contrôler les choses importantes de votre vie? } \\
\text { - Vous êtes-vous senti.e confiant.e dans vos capacités à prendre en main } \\
\text { vos problèmes personnels? } \\
\text { - Avez-vous senti que les choses allaient comme vous le vouliez? } \\
\text { - Avez-vous trouvé que les difficultés s'accumulaient à un tel point que vous } \\
\text { ne pouviez les gérer? }\end{array}$ \\
\hline \multicolumn{2}{|c|}{ Score Mesures de protection pour soi-même (item n. 35) et pour ses enfants (item n. 36) } \\
\hline Classes & 2 classes: "jusqu’à trois protections", "de quatre à huit protections" \\
\hline Enoncés & $\begin{array}{l}\text { - Vous portez des gants } \\
\text { - Vous portez un masque } \\
\text { - Vous portez une combinaison } \\
\text { - Vous vous écartez d'au moins } 1 \text { mètre quand vous croisez quelqu'un à } \\
\text { l'extérieur } \\
\text { - Vous vous lavez les mains plusieurs fois par jour } \\
\text { - Vous vous désinfectez les mains avec du gel hydro-alcoolique } \\
\text { - Vous lavez les vêtements que vous avez portés à l'extérieur du domicile } \\
\text { - Vous désinfectez les poignées de porte, interrupteurs ou autres surfaces } \\
\text { potentiellement contaminées }\end{array}$ \\
\hline \multicolumn{2}{|c|}{ Score Sentiments positifs (item n. 40) } \\
\hline Classes & 3 classes: "entre 0 et 1 sentiment”, " 2 sentiments", " 3 sentiments" \\
\hline Enoncés & «détendu.e», «en forme», «heureux.se» \\
\hline \multicolumn{2}{|c|}{ Score Sentiments négatifs (item n. 40) } \\
\hline Classes & 2 classes : "entre 0 et 3 sentiments", "entre 4 et 6 sentiments" \\
\hline Enoncés & «fatigué.e», «irrité.e», «stressé.e», «triste», «inquièt.e», «angoissé.e» \\
\hline \multicolumn{2}{|c|}{$\begin{array}{l}\text { Score Pression ressentie pendant le confinement concernant la façon de s'occuper de ses } \\
\text { enfants (item n. 44) }\end{array}$} \\
\hline Classes & $\begin{array}{l}3 \text { classes issues de la somme des valeurs attribuées aux réponses "pas du } \\
\text { tout» (ou non concerné.e) (0), «un peu» (1) et «beaucoup» (2) } \\
\text { - "pas ou peu de pression" (scores inférieurs à } 2 \text { ) } \\
\text { - "entre } 1 \text { et } 2 \text { pressions forte }(s) \text { " (scores entre } 2 \text { et } 4) \\
\text { - "plus de deux pressions fortes" (scores supérieurs à } 4)\end{array}$ \\
\hline
\end{tabular}




\begin{tabular}{|c|c|}
\hline Enoncés & $\begin{array}{l}\text { - Si vous êtes en couple, de votre famille ou celle de votre conjoint.e } \\
\text { - Si vous êtes en résidence alternée, de votre ex conjoint.e, de la famille de } \\
\text { votre ex conjoint.e } \\
\text { - De certains membres de la famille (fratrie, parents, oncles, tantes...) } \\
\text { - De vos ami.es ou de vos collègues } \\
\text { - Des éducateurs.trices ou des enseignant.es } \\
\text { - Des médias (journaux, TV...) } \\
\text { - Des réseaux sociaux (forum en ligne, sites spécialisés du Web) } \\
\text { - Des professionnels de santé ou des intervenants sociaux }\end{array}$ \\
\hline \multicolumn{2}{|c|}{ Score Perception du rôle parental durant le confinement (item n. 45) } \\
\hline Classes & $\begin{array}{l}3 \text { classes: } \\
\text { - "perception satisfaisante" («en désaccord» ou «très en désaccord» avec les } \\
4 \text { énoncés négatifs et "d'accord» ou "très d'accord» avec l'énoncé positif) } \\
\text { - "neutre" (comme supra, plus «ni en accord, ni en désaccord» avec au } \\
\text { moins un énoncé) } \\
\text { - "perception non satisfaisante" (au moins une réponse «d'accord» ou «très } \\
\text { d'accord» aux énoncés négatifs ou «en désaccord» ou «très en désaccord» } \\
\text { à l'énoncé positif }\end{array}$ \\
\hline Enoncés & $\begin{array}{l}4 \text { énoncés négatifs: } \\
\text { - J'ai l'impression que je n'en fais pas assez pour stimuler ou éduquer mes enfants } \\
\text { - J'ai l'impression qu'on me demande de faire des activités éducatives avec } \\
\text { mes enfants pour lesquelles je ne me sens pas compétent.e } \\
\text { - Je crains que mes enfants souffrent de manière durable du manque de } \\
\text { stimulation ou d'activités éducatives } \\
\text { - Je me sens dépassé en face à toutes les choses que je dois faire pour m'oc- } \\
\text { cuper de mes enfants } \\
1 \text { énoncé positif: } \\
\text { - Je me dis que je suis la personne la mieux placée pour savoir ce qui est } \\
\text { bon pour mes enfants }\end{array}$ \\
\hline \multicolumn{2}{|c|}{ Score Désagréments subis dans le logement lieu du confinement et dans son environnement (item n. 58) } \\
\hline Classes & 3 classes: "aucun problème", " 1 problème", "entre 2 et 8 problèmes" \\
\hline Enoncés & $\begin{array}{l}\text { - Pollution à l'extérieur du logement } \\
\text { - Manque de sécurité à l'extérieur du logement } \\
\text { - Saleté des rues ou du quartier } \\
\text { - Difficultés à trouver certains produits alimentaires de première nécessité } \\
\text { - Nuisances sonores à l'extérieur du logement (bruit de la rue, des voisins) } \\
\text { - Tensions ou conflits avec des voisin.es. } \\
\text { - Nuisances sonores à l'intérieur du logement (bruit des autres occupant. } \\
\text { es du logement) } \\
\text { - Tensions ou conflits avec des occupant.es du logement } \\
\text { - Manque de place à l'intérieur du logement } \\
\text { - Aucun de ces problèmes }\end{array}$ \\
\hline
\end{tabular}

Annexe 1 - Scores créés à partir des items du questionnaire. 


\section{Références bibliographiques}

Almeida M., Shrestha A.D., Stojanac D., Miller, L.J. (2020): The Impact of the COVID-19 Pandemic on Women's Mental Health. Archives of Women's Mental Health, 23(6), pp. 741-748 (https://doi. org/10.1007/s00737-020-01092-2; date de dernière consultation: 22.12.20).

Bonnéry S., Douat É. (dir.) (2020): L’éducation aux temps du coronavirus. Paris: La Dispute.

Bessière C., Biland E., Gollac S., Marichalar P., Minoc J. (2020): Penser la famille aux temps du COVID-19. Mouvements vol. 8 (https://hal.archives-ouvertes.fr/hal-02954508; date de dernière consultation: 15.02.21).

Bugeja-Bloch F., Crepin L. (2020): Une double peine: les conditions de logement et de confinement des familles monoparentales. Métropolitiques, 4 Juin, pp. 1-6 (https://metropolitiques.eu/Unedouble-peine-les-conditions-de-logement-et-de-confinement-des-familles.html; date de dernière consultation: 22.12.20).

Choi S., Byoun S.-J., Kim E.H. (2020): Unwed Single Mothers in South Korea: Increased Vulnerabilities during the COVID-19 Pandemic. International Social Work, n. 63, pp. 676-680 (https://doi. org/10.1177/0020872820941040; last access: 09.02.21).

Lambert A., Cayouette-Remblière J., Guéraut E., Bonvalet C., Girard V., Le Roux G., Langlois L. (2020): Logement, travail, voisinage et conditions de vie: ce que le confinement a changé pour les Français. Note de Synthèse n. 10, Vague n. 6, INED (https://www.ined.fr/fichier/rte/General/ ACTUALIT\%C3\%89S/COVID19/note-synthese-Cocovi-finale.pdf; date de dernière consultation: 15.02.21).

Marchetti D., Fontanesi L., Mazza C., Di Giandomenico S., Roma P., Verrocchio M.C. (2020): Parenting-Related Exhaustion During the Italian COVID-19 Lockdown. Journal of Pediatric Psychology, n. 45, pp. 1114-1123 (https://doi.org/10.1093/jpepsy/jsaa093; date de dernière consultation: 22.12.20).

Modak M., Zaouche Gaudron C., Séraphin G. (2021-à paraître): L'accueil du jeune enfant des parents en situation de pauvreté: quelles vulnérabilités? Cahier du CEIDEF.

Neyrand G., Rossi P. (2007): Monoparentalité, précarité et femme sujet. Toulouse: Erès.

Okabe-Miyamoto K., Folk D., Lyubomirsky S., Dunn E.W. (2021): Changes in Social Connection during COVID-19 Social Distancing: It's not (Household) Size that Matters, it's Who You're With. PLOS ONE, n. 16, e0245009 (https://doi.org/10.1371/journal.pone.0245009; dernière consultation: 9.2.21).

Paillé P., Mucchielli A. (2016): L'analyse qualitative en sciences humaines et sociales. Paris: Armand Colin.

Paugam S. (2000): Le salarié de la précarité. Paris: PUF.

Rabier R. (2014): Les familles monoparentales, souvent en situation de précarité. Insee Analyses, n. 2 (https://www.insee.fr/fr/statistiques/1285832; date de dernière consultation: 22.12.20).

Thierry X., Geay B., Pailhé A., Berthomier N., Camus J., Cauchi-Duval N., Solaz A. (2021): Les enfants à l'épreuve du premier confinement. Population \& Sociétés, n. 585, pp. 1-4 (https://www.ined.fr/ fr/publications/editions/population-et-societes/enfants-a-epreuve-du-premier-confinement/; date de dernière consultation: 16.04.21).

Tso W.W.Y., Wong R.S., Tung K.T.S., Rao N., Fu K.W., Yam J.C.S., Chua G.T., Chen E.Y.H., Lee T.M.C., Chan S.K.W., Wong W.H.S., Xiong X., Chui C.S., Li X., Wong K., Leung C., Tsang S.K.M., Chan G.C.F., Tam P.K.H., Chan K.L., Kwan M.Y.W., Ho M.H.K., Chow C.B., Wong I.C.K., Lp P. (2020), Vulnerability and Resilience in Children during the COVID-19 Pandemic. European Child \& Adolescent Psychiatry, November, n. 17, pp.1-16 (https://doi.org/10.1007/s00787020-01680-8; last access: 22.12.20).

Vanderschelden M. (2014): Les Ruptures familiales. État des lieux et propositions. Rapport au Haut Conseil de la Famille (https://www.vie-publique.fr/sites/default/files/rapport/pdf/144000594.pdf; date de dernière consultation: 22.12.20).

Zaouche Gaudron C. (2017): Enfants de la précarité. Toulouse: Erès. 
Zaouche Gaudron C., Boyer D. (2021): Accueil des jeunes enfants en situation de pauvreté. Le Furet, n. 100 , mars, pp. 34-35.

Zhou M., Hertog E., Kolpashnikova K., Kan M.-Y. (2020): Gender Inequalities: Changes in Income, Time Use and Well-Being before and during the UK COVID-19 Lockdown. SocArXiv (Preprint), pp. 1-16 (https://doi.org/10.31235/osf.io/u8ytc; last access: 14.02.21). 